\title{
Propaganda und die Welt der Videospiele aus Sicht des Jugendschutzes
}

\section{Saskia Lanser/Britta Schülke}

Die Welt der Videospiele bietet für die unterschiedlichsten Menschen und Persönlichkeiten einen Raum, sich auszuleben. $\mathrm{Ob}$ über Konsole, PC oder Tablet - zunächst ist sie allen zugänglich, die über die technischen Grundvoraussetzungen verfügen. Für viele Kinder und Jugendliche machen Videospiele, auch eingebettet in Spiel-Communities, einen festen Bestandteil des Alltages aus. Es werden Spiele gespielt, (Spiel-)Erfahrungen geteilt, Charakterprofile erschaffen und gepflegt. Doch was ist, wenn man dort - bildlich oder in Textform - auf Gedankengut trifft, durch das Menschen gezielt ausgegrenzt oder abgewertet werden?

Insbesondere im Online-Bereich kann es leicht passieren, dass man über problematische oder gar strafrechtlich relevante Inhalte stolpert. Es reichen ein, zwei Klicks, um über eine Kommentarleiste auf Gruppen oder Spieler*innenprofile zu gelangen, bei denen extremistisches, kriegsverherrlichendes oder menschenverachtendes Gedankengut eindeutig erkennbar ist. Einerseits verdeutlicht dies, dass sich in virtuellen Räumen dieselben Weltanschauungen wiederfinden wie im Analogen auch. Andererseits handelt es sich jedoch nicht nur um individuelle und vereinzelte Bekenntnisse, und es ist ungenügend und fatal, solche Inhalte im Netz als »freie Meinungsäußerung« abzutun.

Die Intention, Videospiele zu Anwerbeund Rekrutierungszwecken oder einer ideologischen Festigung zu nutzen, stellt kein neuartiges Phänomen dar. Bereits Ende der 80er-Jahre wurde sichtbar, dass in Deutschland Anhänger*innen aus dem rechtsextremen Spektrum das Medium Videospiel und allgemein virtuelle Räume für sich zu nutzen versuchten (siehe z.B. die KZ-ManagerReihe). Auch gegenwärtig werden Internetforen oder virtuelle (Spiel-)Gemeinschaften gezielt für die Verbreitung extremistischer Inhalte genutzt. Unbeteiligte können ungewollt mit ihnen konfrontiert werden (z.B. durch Online- oder Browserspiele), in manchen Fällen kann auch das Spielgeschehen beeinträchtigt werden (wie z.B. bei dem Survival-Game Rust ${ }^{1}$ ). Eine unkomplizierte Ansprache zeigt sich so auch in Charakterprofilen oder losen Spielgemeinschaften, deren ideologische Verortung unverkennbar ist. ${ }^{2}$ Bekannte Muster werden aufgegriffen, in einen anderen Kontext gesetzt und zielgruppenorientiert kommuniziert. Spielinterne Logiken können auf gegenwärtige gesellschaftliche Umstände angewandt werden. Ähnlich der Thematik rund um Wirkungstheorien zu gewalthaltigen Videospielen fehlen gegenwärtig Studien, ob und wie Videospiele mit propagandistischen Inhalten überhaupt zu einer eindeutigen Ideologisierung beitragen könnten. Die Thematik wird dadurch aber nicht irrelevant, denn die Welt der Videospiele ermöglicht einen besonderen Zugang zu Jugendlichen und extremistische Gruppierungen verbreiten ihre Ansprachen im Netz über die unterschiedlichsten Formate. Wie bei anderen Medienformaten auch stellt die Kombination von Videospielen und Propaganda nur einen Aspekt dar, dem Augenmerk geschenkt werden sollte. Unabhängig ihrer schlussendlichen Wirkung auf einzelne Individuen werden problematische oder sogar strafrechtlich relevante Bilderwelten kommuniziert, festgelegt und ein identitäres (Nicht-)Zugehörigkeitsempfinden provoziert. Die Vermengung von analoger und vir-

Beide Autorinnen arbeiten bei der Arbeitsgemeinschaft Kinder-und Jugendschutz (AJS) NRW e.V. Saskia Lanser ist Musikwissenschaftlerin und Islamwissenschaftlerin (M.A.), Britta Schülke (Volljuristin) ist Justiziarin und Referentin für Kinder- und Jugendschutzrecht.

tueller Realität ist hierbei insofern kritisch, wenn die Ernsthaftigkeit bestimmter Umstände abgeschwächt wird oder für gewisse Altersgruppen nicht nachvollziehbar ist. Einzelne Spielequalitäten, bspw. eine anspruchsvolle und komplexe Grafik, müssen dafür nicht unbedingt im Vordergrund stehen, denn die soziale Komponente vom Phänomen Videospiel bietet äußerst günstige Kommunikations- und Vernetzungsmöglichkeiten, die über ein einzelnes Spielerlebnis hinausgehen. Interessierten oder Sympathisant*innen wird nicht nur eine virtuelle Projektionsfläche sondern ebenfalls eine vereinfachte Kontaktaufnahme zu Personen in ein ideologisches Spektrum hinein geboten. Die jugendaffine Gestaltung und Verbreitung von Propaganda entspricht einer gezielten und gängigen Strategie unterschiedlicher Akteure. ${ }^{3}$ Die Notwendigkeit, sich mit Propaganda in der Welt der Videospiele zu befassen, erschließt sich also über unterschiedliche Aspekte.

\section{Propaganda gleich Propaganda?}

Zunächst ist der Propaganda-Begriff umstritten und nicht einheitlich defi- niert. ${ }^{4}$ Einigkeit herrscht darüber, dass Propaganda zum einen den willentlichen und erkennbaren Versuch darstellt, Meinungen und Ansichten zu reproduzieren und/oder entlang ideologischer Grundsätze zu manipulieren. Zum anderen wird eine Konsequenz beabsichtigt, nämlich die Reaktion auf ebensolche Inhalte, die idealtypisch in bestimmten Denk- und Handlungsweisen resultieren soll. ${ }^{5}$ Ebenso kann von einer »ideologischen Kommunikation « gesprochen werden. ${ }^{6}$ Befasst man sich mit Propaganda in Videospielen, steht vor allem die strafrechtliche Relevanz im Vordergrund. Klar erkennbar ist sie meist durch die Verwendung von Symbolen verbotener Organisationen, die durch ideologische Narrative und die Bedienung eindeutiger Bilderwelten ergänzt werden.

Aber:

- Propaganda funktioniert nicht auf einem kausalen empfangsorientierten Wirkungsprinzip, d.h. die Konfrontation mit Propaganda führt nicht zwangsläufig oder situationsunabhängig zu einer möglichen Ideologisierung entlang der kommunizierten Inhalte.

- Propaganda kann nicht aus dem Nichts heraus agieren und völlig neue Themen platzieren, sondern sie muss an Stimmungen und Themen anknüpfen, diese bündeln und zuspitzen. ${ }^{7}$

- Eine Verbreitung von Propaganda bedeutet nicht, dass es sich um erfolgreiche Propaganda handeln muss.

Verschiedene Konzepte und Erklärungsansätze aus Kommunikations-, Medien-, oder Kulturwissenschaft verweisen auf den Umstand, dass Propaganda dann erfolgreich ist, wenn sie Bedürfnisse von Empfänger*innen aufgreift und »authentische Interessen `von unten $<$ vertritt. $\aleph^{8}$ Im Klartext bedeutet das, dass Spiele mit extremistischen Inhalten für die Masse der Spielenden völlig uninteressant sein können und es gemeinhin auch sind. Szenegebunden können sie aber spielerische »Tabubrüche« mit ideologiestützender Funktion widerspiegeln oder auch auf Netzwerke hinweisen, über die ein Bezug möglich ist. ${ }^{9}$ Die Verbreitung bestimmter Spiele oder Spielmodifikationen (Mods) sollten deshalb auch als Teil klassischer Anwerbung oder Beziehungsarbeit gewertet werden, insbesondere wenn hierfür Kontakte oder Netzwerke (z.B. durch Foren, Gruppenchats) notwendig sind. 
Kommunikations- und Sendungsbewusstsein

Hinsichtlich der Verbreitung von Propaganda aus extremistischen Spektren ist es nötig, sich auch weiter gefasst mit der Welt der Videospiele und ihren Kommunikationsmöglichkeiten auseinanderzusetzen. Medienwirksam gelang es in den letzten Jahren dem sogenannten »Islamischen Staat« (IS), gezielt das Medium Videospiel für eigene Propagandazwecke zu nutzen. Neben eigens produzierten Android-Apps mit dschihadistischer Ästhetik, in denen spielerisch Lesen, Rechnen und Schreiben in arabischer Sprache geübt werden, produzierte der IS Memes, Gifs und Propagandavideos mit Videospiel-Referenzen..$^{10}$ Es wurden nicht nur bekannte Spielreihen entlang ihrer Titel verfremdet (z.B. Call of Duty zu »Call of Jihad « oder Grand Theft Auto - Salil al Sawarim), sondern Ausschnitte bekannter Spiele für eigene Propagandavideos verwendet und/ oder modifiziert. ${ }^{11}$ Diese Strategie ist nicht unbedingt verwunderlich, denn der IS setzte bereits früh auf die gezielte Anwerbung junger Menschen. Auch die sogenannte »Identitäre Bewegung (IB) setzt auf Games: Es geht nicht um simplen Spielspaß, wenn auf Spieleplattformen offizielle IB-Gruppen politische Inhalte vertreten oder ein Let'sPlay-Video zu einem Flashplayer-Spiel gegen den Globalen Pakt für eine sichere, geordnete und reguläre Migration (der sog. »UNMigrationspakt «) veröffentlicht wird. ${ }^{12}$

Eine weitere Dimension stellt die Übertragung des Terroranschlags von Halle 2019 bei der unter Gamer*innen beliebten Streaming-Plattform Twitch dar. ${ }^{13}$ Laut Aussage von Twitch wurde der Stream von etwa 2.200 Personen in den 30 Minuten, bevor er von der Plattform gelöscht wurde, angeschaut. ${ }^{14}$ Der Live-Stream tauchte in keiner Empfehlungsliste auf und lief über einen neu erstellten Account ohne Follower*innenzahlen. Im Nachgang zeigte sich, dass die hohe Zuschauerzahl dadurch zu erklären ist, weil ein Link zum Stream in kürzester Zeit bei unterschiedlichen rechtsextremen Telegram- und Whatsapp-Gruppen geteilt wurde. Die Videoaufzeichnung der menschenverachtenden Gewalttat war - auch bezogen auf die Ansprache und Äußerungen des Attentäters also nicht allgemeinhin öffentlichkeitswirksam, sondern für eine bestimmte Zielgruppe gedacht. Ein solches Sendungsbewusstsein auf öffentlich einsehbaren Plattformen, die im Kontext von Videospielen nicht mehr wegzudenken sind, muss auch zukünftig stärker berücksichtigt und entlang veröffentlichter Inhalte ernst genommen werden.

\section{Strafrechtliche Relevanz von Propaganda}

Auf den ersten Blick mag nicht immer erkennbar sein, wo in einem Spiel Propa- ganda anfängt und wann ein juristisches Einschreiten nötig ist. § 86a StGB regelt zunächst die Verbreitung und Verwendung von Symbolen verbotener Parteien oder Vereinigungen. Ebenfalls ist der Kontext der Verwendung ausschlaggebend (Prüfung der sogenannten Sozialadäquanzklausel gem. § 86 Abs. 3, § 86a StGB) oder ob Volksverhetzung nach $\S 130$ StGB greift, bei dem der Angriff auf die Menschenwürde definiert wird.

So ist § 130 StGB in der Praxis überwiegend ein Delikt Rechtsradikaler, die u.a. mit die Menschenwürde Anderer verletzender Hetze oder der Leugnung bzw. Verharmlosung von NS-Verbrechen auftreten. Die Gefährlichkeit dieser Propaganda liegt in dem hohen Drohungs- und Aufforderungsgehalt bei gleichzeitiger Verrohung und Abstufung gegenüber Verletzungen und Leiden von Angehörigen als minderwertig dargestellter Gruppen. ${ }^{15}$ Vor allem § 130 Abs. 2 StGB muss hier hervorgehoben werden, nach dem die Verbreitung von Medien mit volksverhetzendem Inhalt explizit auch mittels Telemedien strafbar ist. ${ }^{16}$

Häufig zeigt sich in Spielen, die eine propagandistische Funktion erfüllen, dass neben eindeutigen Symbolen eben auch volksverhetzende Aussagen, Gewaltdarstellungen oder Relativierungen ebensolcher vorhanden sind. Trotzdem unterliegt die Wertung um Propaganda nicht ohne Grund immer einer Einzelfallentscheidung, denn Spiele können entlang ihrer Inhalte sehr unterschiedliche Kontexte herstellen. In Bezug auf Ahndungen kann unter Umständen eine Problemlage vorliegen, wenn (neue) Verbote von Symbolen oder die Zugehörigkeit dieser in einen extremistischen Kontext unbekannt sind oder Spiele historische Umstände aufgreifen. Es stellt sich nämlich durchaus die Frage, ob alle Kennzeichen, Akronyme oder Zahlencodes bspw. aus dem rechtsextremen Spektrum - auch in abgewandelter Form - von Unbeteiligten immer eindeutig zugeordnet werden können. Deshalb ist die seit August 2018 auf Videospiele angewandte Sozialadäquanz durch die USK ein zu begrüßender Schritt, da trotz der Verwendung verbotener Symbole ein eindeutiger Unterschied zwischen Spielen mit propagandistischen Inhalten und Spielen, die sich reflektiert einem historischen Zeitgeschehen widmen, festgemacht werden kann. Ihre Anwendung ermöglicht den bewussten und kritischen Umgang mit bestimmten Symbolen.

\section{Beispiel für Grauzonen: Das Videospiel »IS Defense“}

IS Defense von Destructive Creations kann als Beispiel dafür dienen, welche Grauzonen Videospiele bedienen können. Zunächst handelt es sich um einen TowerDefense-Shooter, in dem aus der Rolle eines NATO-Soldaten heraus einfallende Truppen des sogenannten Islamischen Staates (IS) an Europas Grenzen - bzw. Stränden - bekämpft werden. Dem Spielszenario nach hat der IS im Jahre 2020 das gesamte nördliche Afrika erobert, von dem aus er Europa über den Mittelmeerweg zu erobern versucht. Entlang eines Shoot-Em-Up-Prinzips werden somit Bereiche vor einfallenden Massen an ISKämpfern verteidigt. Zunächst könnte diese Ausgangslage suggerieren, dass es sich um einen Shooter handelt, der eben ein explizites Feindbild beinhaltet. Problematisch ist jedoch die Bilderwelt, in die das Spielgeschehen eingebettet ist: Die Verteidigung von Europas Stränden knüpft stark an rechtsextreme Forderungen zu der Situation vieler Bootsübersetzungen von geflüchteten Menschen über die Mittelmeerroute an. Das Narrativ, Europa sei als »letzte Bastion« vor »einfallenden Massen an (IS-)Terroristen « zu verteidigen, geht mit einer stereotypen sowie de-humanisierenden Darstellung der zu erschießenden Gegner und einer intendierten Glorifizierung der eigenen Aufop- ferung für diese Mission einher (Zitat der Spielemacher*innen: »The player takes the role of NATO's stationary machine-gun operator, deployed to defend the shores of Europe. His task is to blast as many of the invaders as possible, until his glorious death«).*) Bei seinem Spiele-Vorgänger "Hatred « stand das Entwickler*innen-Studio um seinen CEO Jarosław Zieliński bereits in der Kritik, anti-islamische Einstellungen zu vertreten, woraufhin eine offizielle Erklärung auf der Website von Destructive Creations folgte.**) Selbst wenn dem Entwickler*innenteam eine gezielte Provokations-Marketingstrategie unterstellt werden kann, werden die dargelegten Bilderwelten nicht abgemildert und es bleibt die Frage im Raum, weshalb ausgerechnet dieses Spielszenario kreiert wurde. Allein die Kommentarspalten auf Steam bspw. verdeutlichen, dass die Attraktivität des Spiels für manche Kommentator*innen nicht nur in dem günstigen Preis und einer flüssigen Spielmechanik liegt.

*) Zitat aus der offiziellen Spielebeschreibung von IS Defense auf der Spielplattform Steam.

**) Nachzulesen unter https://www.polygon. com/2014/10/20/7020277/ceo-behindhatred-neo-nazi-anti-islamic-responds bzw. https://destructivecreations.pl/inny -wpis-2/ 
Schwieriger ist, wenn Spielinhalte für extremistische Ansichten bzw. Tendenzen besonders attraktiv erscheinen, aber keine direkte Verbindung zu verfassungswidrigen Organisationen oder entsprechender Symbolik existiert. Die Verwendung problematischer Charakterdarstellungen und undifferenzierter Storylines mit Realitätsoder Gegenwartsbezug können zwar eine propagandistische Grundtendenz stützen (siehe z.B. IS Defense) oder eine geschichtsrevisionistische Komponente ermöglichen (siehe z.B. Frontline: Road to Moscow ${ }^{17}$ ), erfüllen aber aus nachvollziehbaren Gründen keinen Straftatbestand. Trotzdem ist hierbei auffällig, dass sie sich aufgrund ihrer Anlage und Anschlussfähigkeit an ideologische Narrative häufig großer Popularität in rechtsextremen Kreisen erfreuen. ${ }^{18}$ Interessanterweise zeichnen sich solche Spiele oft auch durch einen geringen Preis aus, was für viele junge Spieler*innen einen unbedarften Anreiz darstellen kann, diese kostengünstig »auszuprobieren«.

\section{Jugendschutz in Spielen}

Spiele in Deutschland werden neben den strafrechtlichen Normen aus jugendschutzrechtlicher Sicht geprüft, da sie die gesunde Entwicklung von Kindern und Jugendlichen beeinträchtigen oder sogar gefährden können. Das Jugendschutzgesetz unterscheidet hier zwischen entwicklungsbeeinträchtigenden und jugendgefährdenden Medien. Zumindest Spiele auf Trägermedien müssen vor Verkaufsstart gem. § 14 JuSchG ein Altersfreigabeverfahren mit den offiziellen Alterskennzeichen durchlaufen. Der Gesetzgeber hat an dieser Stelle ein System der "regulierten Selbstregulierung «, das heißt eine Selbstregulierung, die in ein hoheitlich, rechtlich abgesichertes Kontrollsystem eingebettet ist, installiert, damit die wertgebundene »regulative Idee« des Jugendschutzes zeitgemäß auch für Trägermedien angewendet werden kann. Durchgeführt werden diese Altersfreigabeverfahren von der USK (Unterhaltungssoftware Selbstkontrolle - die freiwillige Selbstkontrolle der Computerspielewirtschaft), die die Spiele dann je nach Inhalt mit der jeweiligen Altersfreiga-

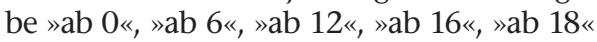
kennzeichnet. Filme und elektronischeSpiele ohne Kennzeichen dürfen nicht an Kinder und Jugendliche (Minderjährige / unter 18-Jährige) abgegeben werden. Als jugendgefährdend eingestufte Medien dürfen nicht in den Verkehr gebracht werden und unterliegen besonderen Regelungen. Strafrechtliche Indikatoren für eine Indizierung durch die Bundesprüfstelle für jugendgefährdende Medien (BPjM) sind u.a. Volksverhetzung, Kriegs- oder Gewaltverherrlichung, die unter $\S \S 86,130,130 a$, oder 131 des
Strafgesetzbuches fallen. Spiele, die extremistische Propaganda enthalten, landen somit auf dem Index.

Telemedien, die ausschließlich online existieren, unterliegen bisher keiner gesetzlichen Kennzeichnungspflicht. Zwar können Anbieter*innen ihre Online-Spiele von der USK nach dem IARC-Verfahren mit freiwilligen Altersfreigabeempfehlungen versehen, aber eine vergleichbare gesetzliche Vorlagepflicht wie für Trägermedien besteht nicht. Online-Spiele sind häufig auch in benachbarten Ländern oder im außereuropäischen Raum angesiedelt (gehostet) und dadurch aufgrund des Territorialprinzips einem möglichen Zugriff deutscher Behörden entzogen. Dennoch wird in Deutschland die strafrechtliche Relevanz von Inhalten im Netz durch verschiedene Institutionen geprüft (z.B. durch die Kommission für Jugendmedienschutz [KJM] oder jugendschutz.net). Finden sich im Internet etwa Inhalte, die gegen geltende Normen verstoßen und/oder jugendgefährdend sind, werden je nach Sachlage Anbieter*innen bzw. Plattform-Betreiber*innen kontaktiert und weitere Schritte eingeleitet. Die dezentrale Struktur des Internet erschwert eine solche Arbeit, weshalb auch Meldungen und Verweise auf ebensolche Inhalte eine wichtige Unterstützung darstellen.

Im grenzüberschreitenden WorldWideWeb ist der Wirkungskreis hiesiger Normen aber faktisch begrenzt. Eine zusätzliche Möglichkeit wäre die stärkere Durchsetzung von Alterskennzeichen bei OnlineSpielen, da die Prüfkriterien eine inhaltliche Einordnung und Orientierung bieten. Die Zusammenarbeit der USK mit der IARC (International Age Rating Coalition) ist in Bezug auf Telemedienspiele eine sinnvolle Ergänzung, insbesondere vor dem Hintergrund, dass das IARC-Verfahren ein einheitliches, globales System zur weltweiten Altersbewertung von online vertriebenen Games ist und alle an dieses System angeschlossenen Vertriebsplattformen damit Alterskennzeichen der USK verfügbar machen. $\mathrm{Zu}$ den angeschlossenen Systemen zählen bislang: Microsoft Windows Store, Xbox Store, Google Play Store, Nintendo eShop und der Oculus Store. Da das Verfahren mangels gesetzlicher Grundlagen nicht verpflichtend ist, hängt der Prozess vom freiwilligen, eigenverantwortlichen Handeln der Anbieter*innen ab. Positiv ist, dass das IARC-System die Überprüfung verwaltungstechnisch vereinfacht und in Deutschland von der USK überwacht wird. IARC-Einstellungen werden auf Beschwerden von Nutzer*innen hin überprüft, wodurch die Richtigkeit der Kennzeichen nach etablierten Standards gewährleistet wird. Große am Markt agierende Anbieter, deren Basis-Versionen der Spiele in Deutschland auch auf Trägermedien verfügbar sind und demnach ohnehin geprüft werden, beteiligen sich so gut wie flächendeckend am IARC-System. Aber auch reine Online-Spiele-Anbieter, wie das derzeit bei Kindern und Jugendlichen beliebte "Fortnite " beteiligen sich, um die Akzeptanz des Spiels bei Erziehenden zu erhöhen. Weniger zu erwarten ist, dass Anbieter*innen von Spielen mit propagandistischen Inhalten Wert auf eine ebensolche Prüfung legen. Spiele mit extremistischen Inhalten, ob offiziell indiziert oder nicht, werden wohl auch zukünftig konspirativ und entlang nicht-öffentlicher Kanäle geteilt. Diesen Umstand zu unterbinden ist mühselig und scheint aufgrund der schieren Menge entsprechender Angebote aussichtslos. Viel wichtiger ist deshalb, dass solche nicht flächendeckend oder selbstwirksam auf frei zugängigen Plattformen verbreitet werden können und Unbeteiligte ungewollt mit ihnen konfrontiert werden. Denn häufig beinhaltet Propaganda nicht nur eine Ansprache für eine bestimmte Gruppe, sondern gleichzeitig die Abwertung und Ausgrenzung einer anderen Gruppe. In Bezug auf Charakterprofile, Kommentarfunktionen oder Foren gilt es, dass in Community-Standards inhaltlich Position bezogen werden muss und diese konsequent durchgesetzt wird.

\section{Fazit}

Forderungen nach strikteren und ausgeweiteten staatlichen Strukturen auf den Online-Bereich, Regulierungsmöglichkeiten und -maßnahmen inklusive, erscheinen als Reaktion auf hiermit zusammenhängenden Problemlagen durchaus nachvollziehbar. Neue Gesetze und Kontrollmechanismen können in Bezug auf Verbreitungsmöglichkeiten problematischer Inhalte durchaus vermeintliche Sicherheit suggerieren. Oftmals zeigt sich in der Weiterentwicklung technischer Möglichkeiten jedoch, dass sich Verbreitungskanäle zeitschnell ändern können und sich beobachtende bzw. eingreifende Systeme dementsprechend ständig (mit)anpassen müssen. In Anbetracht der stetigen technischen Weiterentwicklungen braucht es deshalb eine starke Flexibilität und Anpassungsfähigkeit, um Trends oder problematische Entwicklungen abschätzen und mitunter zeitschnell und adäquat reagieren zu können. Bereits jetzt können strafrechtlich relevante Inhalte gemeldet (jugendschutz.net, Internet-BeschwerdeStelle) oder angezeigt werden. Statt überregulatorischer Bestrebungen zeigt der Ansatz der regulierten Selbstregulierung, wie auf die weltweite Verbreitung und Vernetzung von Inhalten reagiert werden kann. Notwendigerweise muss der Kinder- und Jugendschutz dafür globaler aufgegriffen und nicht ausschließlich auf nationaler Ebene ausgehandelt werden. Der Fokus 
sollte darauf liegen, einen gesetzlichen Rahmen wertegebunden auszuarbeiten.

Zudem ist es unerlässlich, Eltern und andere Erziehungsberechtigte besser $\mathrm{zu}$ befähigen, Kinder und Jugendliche vor gefährdenden Einflüssen $\mathrm{zu}$ schützen und sie sensibel für propagandistische Einflüsse zu machen. Dafür muss der PropagandaBegriff im Kontext von Videospielen nicht juristisch erweitert werden. Eine einfache Hilfestellung bietet die Alterskennzeichnung durch die USK, für den Online-Bereich auch in erweiterter Form durch das IARC-System. Ähnlich zu anderen Themen muss aber der Fokus darauf liegen, junge Menschen zu befähigen, sich vor gefährdenden Einflüssen zu schützen und sie zu Entscheidungs- und Kritikfähigkeit, Eigenverantwortlichkeit sowie zur Verantwortung gegenüber ihren Mitmenschen zu führen (\$14 SGB VIII). Hierfür sind medienpädagogische Ansätze, die den Aspekt der Politischen Bildung aufgreifen, unerlässlich. Dazu zählt nicht nur die altersgerechte Förderung von Medienkritikfähigkeit und allgemeine Sensibilisierung, extremistische Inhalte $\mathrm{zu}$ erkennen, sondern ebenfalls die gesellschaftspolitische Auseinandersetzung, wie generell mit solchen Inhalten umgegangen wird. Die Vorstellung, dass junge Menschen durch Propaganda vor allem angesprochen i.S.v. angeworben werden sollen, übersieht oft einen wichtigen Umstand: Viele Menschen sind durch die rassistischen und menschenverachtenden Inhalte betroffen, da sie gemeint sind. Kinder und Jugendliche verstehen oftmals, ob sie gruppenbezogen Zielscheibe eines Kommentars oder geäußerter Inhalte sind. Diese gilt es zu schützen und zu stärken, und das auch in digitalen Räumen.

Das kann mit einem zeitgemäßen Zusammenspiel von gesetzlichem und erzieherischem Jugendschutz gelingen, der sich den aktuellen Herausforderungen stellt und seiner Kompetenzen und Wirkungen bewusst ist. Denn viele Phänomene sind nicht neu, nur die Verbreitungskanäle und Austragungsorte haben sich geändert.

1 Das nur im Mehrplayer-Modus spielbare Rust stand bereits häufiger in der Kritik, rechtsextremen Inhalten (z.B. von Spieler*innen erstellte Gebäude mit nationalsozialistischen Symbolen, virtuelle »Menschenjagden « auf nicht-Weiße Spielcharaktere) zu wenig entgegenzusetzen. Siehe auch Schott, Dom: »Nazis raus aus dem Gaming «, Spieletipps Online vom 21.10.2019 (https://www. spieletipps.de/artikel/10419/1/).

2 Siehe auch Huberts, Christian »Mein Dampf: Die rechtsextreme Parallelwelt auf der Games-Plattform Steam《, veröffentlicht am 26.06.2018 auf DerStandard.de (https://www.derstandard.de/story/ 2000082229261/die-games-plattformsteam-hat-ein-grosses-problem-neonazis).

3 »ugendaffin « bedeutet in diesem Kontext, dass sich Ansprachen visuell an der Ästhetik von bekannten Videospielen orientieren oder eindeutige Videospielreferenzen enthalten. Einen Einblick hierzu liefert https:// www.hass-im-netz.info/.

4 Eine Übersicht über die verschiedenen Forschungsdiskurse, in denen der Begriff Propaganda z.T. unterschiedlich definiert wurde, findet sich in Bussemer, Thymian: Propaganda. Konzepte und Theorien, Wiesbaden 2005, S. 53

5 Vgl. Bussemer, S. 24ff.; Frischlich et al., S. 19f.

6 Vgl. Arnold, S. 64ff.

7 Vgl. Bussemer, S. 24f.

8 Bussemer, S. 25.

9 Vgl. Wittels, S. 25.

10 Jugendschutz.net (Jahresbericht 2016), S. 8 f. und Sonderbericht »Dschihadisten rekrutieren Kinder über Apps«, November 2016 (https://www.jugendschutz.net/fileadmin/ download/pdf/Islamistische_Kinderangebote.pdf).

11 Vgl. Lakomy, S. 9f.; Al-Rawi, S. 4ff; jugendschutz.net (2018), S. 10 u. 14f. Eines der bekannteren Memes des IS stellte das Werbebild von Call of Duty: Black Ops dar, bei dem der Titel in "Call of Jihad: Operation Assad « abgeändert wurde. In Anlehnung an den syrischen Bürgerkrieg wurde die verfälschte Realität um ein Kriegsszenario jugendaffin und mit hohem Wiedererkennungswert verbreitet. In Ergänzung dazu wurde über andere Kanäle des IS die Ansprache kommuniziert, man solle sich »dem echten Call of Duty«, also dem Kriegsgeschehen vor Ort, anschließen.

12 Jugendschutz.net: »Identitäres« Flash-Game gegen den »Migrationspakt«, Dezember 2018 (https://fis.jugendschutz.net/master-detailseite-news/?tx_news pi $1 \% 5$ bnews $\% 5 d=915 \&$ tx news pi1\%5bcontroller\%5d=News\&tx_news_pi1 $\% 5$ baction $\% 5 \mathrm{~d}=$ detail $\& \mathrm{cHash}=0 \mathrm{cf} 7187 \mathrm{f} 12$ fecb2b17c01d3f86563479).

13 Zum Umgang mit dem Tätervideo im pädagogischen Kontext findet sich eine Handreichung von fjp>media (https://www.service stelle-jugendschutz.de/2019/10/umgangmit-dem-taetervideo-aus-halle/).

14 Nachzulesen als Stellungnahme bei Twitter (@Twitch) vom 19.10.2019.

15 Fischer, § 130, S. 1003

16 „Telemedien« beschreibt als Oberbegriff elektronische Informations- und Kommunikationsdienste. Geregelt durch den Jugendmedienschutz-Staatsvertrag (JMStV) fallen deshalb nahezu alle Angebote im Internet - so auch Spiele-Plattformen oder Apps - unter diesen Begriff.

17 In dem Strategiespiel Frontline: Road to Moscow wird aus Sicht der deutschen Wehrmacht der Russlandfeldzug von 1941-43 nachgespielt, mit dem Ziel der erfolgreichen Eroberung Russlands.

18 Vgl. Wittels, S. 24f.

19 Zitat aus der offiziellen Spielebeschreibung von IS Defense auf der Spielplattform Steam.
20 Nachzulesen unter https://www.polygon. com/2014/10/20/7020277/ceo-behindhatred-neo-nazi-anti-islamic-responds bzw. https://destructivecreations.pl/innywpis-2/.

21 Näheres zum Verfahren: http://www.usk. de/iarc/.

\section{Literatur}

Al-Rawi, Ahmed: »Video Games, Terrorism, and ISIS's Jihad 3.0«, in: Terrorism and Political Violence 30 (2018), H. 4, S. 740-760.

Arnold, Klaus: »Propaganda als ideologische Kommunikation«, in: Publizistik 48 (2003), H. 1, S. 63-83.

Bussemer, Thymian: »Psychologie der Propaganda $\ll$ in: APuZ 11 (2007), S. 19-25.

Delwiche, Aaron: From The Green Berets to America's Army: Video Games as a Vehicle for Political Propaganda, in: Williams, J.P./ Smith, J.H. (Hrsg.): The Players` Realm. Studies on the Culture of Video Games and Gaming, Jefferson/N.C. 2007, S. 91-109.

Frischlich, Lena /Rieger, Diana /Morten, Anna: (Hrsg.): Videos gegen Extremismus? CounterNarrative auf dem Prüfstand, hrsg. vom Bundeskriminalamt in Kooperation mit der Forschungsstelle Terrorismus/Extremismus, Wiesbaden 2017.

Fischer, Thomas: Strafgesetzbuch-Kommentar, 63. Aufl., München 2016.

Günther, Thomas: »Das Internet - kein rechtsfreier Raum «, in: Glaser, Stefan /Pfeiffer, Thomas (Hrsg.): Erlebniswelt Rechtsextremismus. Menschenverachtung mit Unterhaltungswert. Hintergründe - Methoden - Praxis der Prävention, Bonn 2013, S. 83-99.

Hall, Stuart: »Kodieren/Dekodieren«, aus dem Engl. übers. v. Bettina Suppelt, in: Hall, Stuart: Ideologie. Identität. Repräsentation. Ausgewählte Schriften 4, hrsg. v. Juha Koivisto u. Andreas Merkens, Hamburg 2004, S. 167-187.

Jäckel, Michael: Medienwirkungen. Ein Studienbuch zur Einführung, 5. überarb. u. erw. Aufl., Wiesbaden 2011.

Jugendschutz.net: Jahresbericht 2016, Mainz 2017.

Jugendschutz.net: 2017 Bericht. Islamismus im Netz, Mainz 2018.

Lakomy, Miron: "Let's Play a Video Game: Jihadi Propaganda in the World of Electronic Entertainment", in: Studies in Conflict \& Terrorism (2017), S.1-24.

Wittels, Malte Switkes vel: »Rechtsextremismus und Revisionismus im Kontext von Computerspielen", in: Amadeu Antonio Stiftung (Hrsg.): Gaming und Hate Speech. Computerspiele in zivilgesellschaftlicher Perspektive, Cottbus 2015, S. 24-26.

Witting, Tanja: : »Wie das Computerspielen Denken und Handeln prägen kann - Erkenntnisse zu Transferprozessen «, in: Fritz, Jürgen (Hrsg.): Computerspiele(r) verstehen. Zugänge zu virtuellen Spielwelten für Eltern und Pädagogen, Bonn 2008, S. 144-162.

Teile dieses Artikels erschienen im AJS Forum 4/2018. (Online abrufbar unter https://www.ajs. nrw.de/forum/ajs-forum-42018/) 